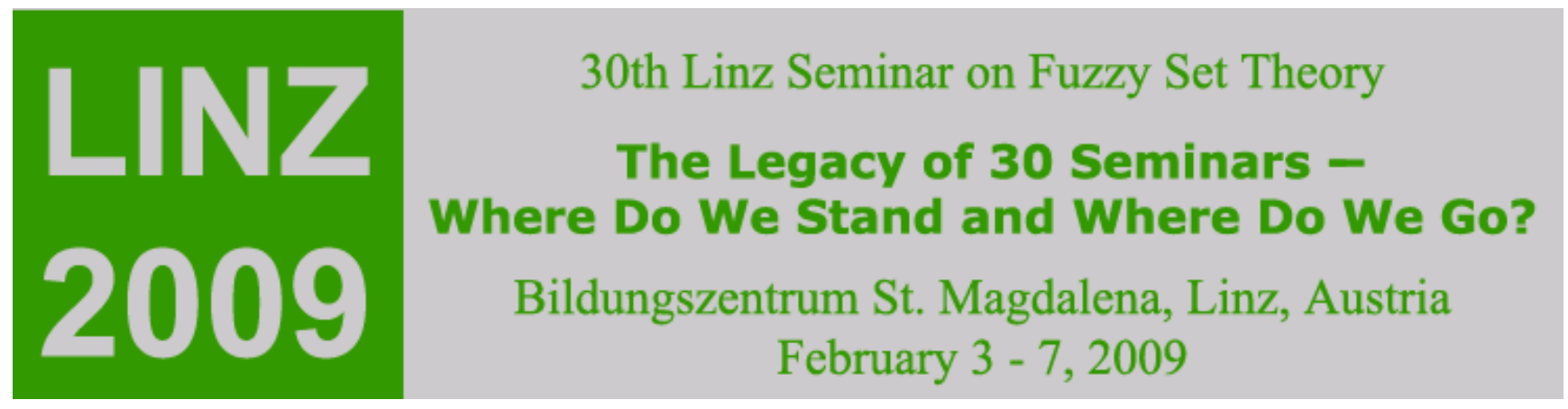

\section{Program Chairs}

Ulrich Bodenhofer

Linz, Austria

Bernard De Baets

Gent, Belgium

Susanne Saminger-Platz

Linz, Austria

\section{Program Committee}

Dan Butnariu

Haifa, Israel

Didier Dubois

Toulouse, France

János Fodor

Budapest, Hungary

Lluis Godo

Barcelona, Spain

Siegfried Gottwald

Leipzig, Germany

Petr Hájek

Praha, Czech Republic

Ulrich Höhle

Wuppertal, Germany

Erich Peter Klement

Linz, Austria

Wesley Kotzé

Grahamstown, South Africa

Radko Mesiar

Bratislava, Slovak Republic

Daniele Mundici

Firenze, Italy

Endre Pap

Novi Sad, Serbia

Stephen E. Rodabaugh

Youngstown, $\mathrm{OH}, \mathrm{USA}$

Lawrence N. Stout

Bloomington, IL, USA

\section{Aldo Ventre}

Napoli, Italy

Siegfried Weber

Mainz, Germany

\section{CALL FOR PAPERS}

Since their inception in 1979, the Linz Seminars on Fuzzy Set Theory have emphasized the development of mathematical aspects of fuzzy sets by bringing together researchers in fuzzy sets and established mathematicians whose work outside the fuzzy setting can provide directions for further research. The philosophy of the seminar has always been to keep it deliberately small and intimate so that informal critical discussions remain central.

LINZ 2009 is the 30th seminar in this series of meetings. Different to previous years, the scope of the seminar does not restrict to a single sub-topic of fuzzy set theory. Instead, the goal is to view fuzzy set theory and the past and future contributions of the Linz seminars from additional perspectives. We want to determine the state of the art achieved within fuzzy set theory, to ask for the impacts on other fields (of mathematics and applications), and to discuss their future research directions and applications.

To pursue the above goals, about $40 \%$ of the seminar will be dedicated to the following kinds of contributions:

1. Reviews (in particular outcomes of the Linz Seminar, contributions to standardizations);

2. Practical applications with profound theoretical foundations in fuzzy set theory (that would not have been possible without);

3. Position papers about future research directions, future applications, and the future of the topic itself.

These contributions should consider one or more of the traditional Linz Seminar subjects:

- Many-valued logics and fuzzy logic in the narrow sense

- Non-classical measures and integrals

- Decision theory and preference modeling

- $\quad$ Logical connectives and aggregation functions

- Algebraic, topological and categorical aspects of fuzzy sets

- Relations to probability theory and statistics

The complementary $60 \%$ of the seminar are devoted to latest research advances in any of the above topics. However, we expect potential contributions to highlight their connection to other mathematical disciplines and/or practical applications. In particular, young researchers ( $\mathrm{PhD}$ students, junior post-docs) are encouraged to submit their latest results.

The following invited speakers (in alphabetic order) have already confirmed their participation:

- Isabelle Bloch, Ecole Nationale Supérieure des Télécommuncations, Paris (France)

- Petr Cintula, Academy of Sciences of the Czech Republic, Prague (Czech Republic)

- Eyke Hüllermeier, Philipps-University Marburg, Marburg (Germany)

- Daniele Mundici, University of Florence, Florence (Italy)

- Dag WesterståhI, University of Gothenburg, Goteborg (Sweden)

The "Linz tradition" has these key features: the number of participants of the Linz Seminars is usually bounded above by 40 with broad international representation and a mix of pure and applied interests; there are no parallel sessions so that all participants focus on each presentation and fully engage each topic; and there is ample time for discussion of each presentation, with follow-up round tables for discussion of open problems and issues raised in the talks.

Please send your extended abstract (1-4 pages)

until November 9, 2008 to linz2009@fllI.jku.at

Further Information:

http://www.flII.jku.at/research/linz2009 